\title{
The world's fastest wireless backhaul radio
}

\section{A case study in industry-research collaboration}

\author{
Dr Rowan Gilmore \\ EM Solutions Pty Ltd \\ Dr Xiaojing Huang \\ CSIRO Digital Productivity and Services (previously) and currently \\ University of Technology Sydney \\ Dr Richard Harris \\ EMClarity Pty Ltd
}

Summary: Fibre is commonly perceived to be the dominant transport mechanism for transferring data from access points back to a central office, where it is aggregated onto the core network. However, high speed and long range wireless backhaul remains a cost-effective alternative to fibre networks. In some areas, wireless backhaul is dominant and becoming more and more attractive. However, commercially available wireless backhaul systems do not meet the requirements for both high speed and long range at the same time with sufficiently low latency for some applications. Traditional microwave systems can achieve long transmission range, but the data rates are then limited to a few hundred megabits per second. Multi-gigabit per second wireless communications can be achieved using millimetre-wave (mm-wave) frequency bands, especially in E-band, but the practical transmission range has then always been a major weakness.

In this article, the world's first 5 Gbps radio solution - and the fastest commercial backhaul product developed by EM Solutions Pty Ltd with the Commonwealth Scientific and Industrial Research Organisation (CSIRO) - is described. As well as achieving a state-of-the-art data rate, other key design features include maximal path length, minimal latency, and constant antenna pointing under wind and tower vibration.

\section{Introduction}

According to the Cisco visual networking index (VNI) global mobile data traffic forecast (Cisco), global mobile data traffic reached 1.5 Exabytes ( 1 Exabyte $=2^{60}$ bytes) per month at the end of 2013, and is expected to grow 10 times by 2018, a compound annual growth rate (CAGR) of 61 percent (Cisco 2014). The rapid increase in mobile data traffic shown in Figure 1 is mainly due to the widespread deployment of broadband wireless access services enabled by smart phones and tablets, as well as next generation networks, such as the worldwide interoperability for microwave access (WiMAX) ( $\mathrm{Fu}$ et al. 2010: 50 - 58) and fourth 
generation long term evolution (LTE)- Advanced systems (Fodor et al. 2011: 84 - 91), which can offer data rates from hundreds of Megabits per second (Mbps) to 1 Gigabit per second (Gbps). These high-speed access networks are attractive alternatives to wired access networks such as fibre optic networks, and provide broadband services in a cost-effective manner. In the meantime, network operators are also seeking solutions to support low latency applications, such as real-time machine-to-machine communications, interactive multiplayer gaming, high frequency trading, and cloud computing (Transmode).

\section{Exabytes per Month}

\section{$61 \%$ CAGR 2013-2018} 18

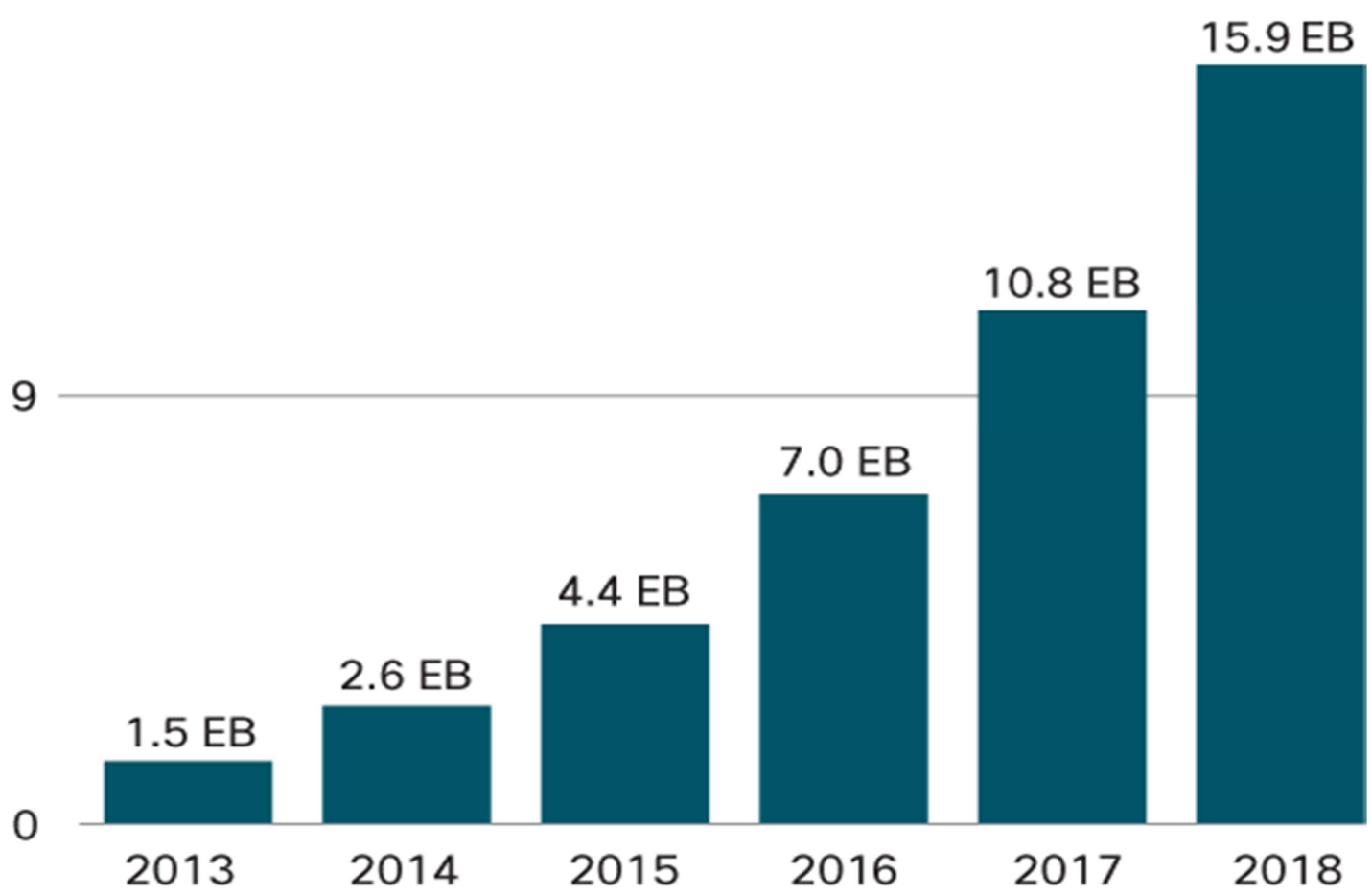

Figure 1: Cisco forecasts mobile data traffic of 15.9 Exabytes per month by 2018 (Cisco 2014)

Due to the ever-growing capacity required to support high speed broadband services, the backhaul network, which transfers data traffic from cell sites of a wireless access network to the core network or a switching centre, is under intense pressure.

There are a number of challenges to implement such backhaul. The first is how to achieve higher data rate or capacity up to multiple Gigabits per second. For example, if the capacity of a cell (or sector) in a broadband wireless access (BWA) base station is $1 \mathrm{Gbps}$, the backhaul capacity required by a three sector base station would be at least 3 Gbps. Sometimes the traffic from multiple base stations will be aggregated together across a backhaul link before reaching the core network. This will drive the backhaul capacity to a much higher rate, say 10-15 Gbps. 
The second challenge is the link distance of the backhaul. To deliver BWA services to unserved areas, such as rural and regional areas that are often quite remote from the main telecommunication infrastructure, a long distance backhaul link is required. In countries with large geographical areas of low population density, such as Australia, long-distance high-data rate wireless backhaul links are essential to bring broadband services to the last $10-30 \%$ of the population. Of course, the data rate challenge is not as great in rural areas because of the sparsity of users, but over time this too may become an issue.

The third challenge is how to achieve low latency communications between end users across the backhaul networks. Although low latency has always been important for delivering high quality voice, video and data services in broadband networks, recent application requirements within many industry sectors, such as gaming and finance, have made low latency an even more important consideration in data transport. It becomes even more critical as the intelligence within the network is centralised to reduce costs, and functions previously delivered at the access node must now be performed within the core network.

Fibre is the obvious solution - and indeed the primary medium - to deliver leased synchronous digital services and Ethernet services. It is the first choice for high data rate backhaul at speeds from $155 \mathrm{Mbps}$ up to 10 Gbps. However, due to its high installation expense, owning a fibre is a significantly capital-intensive (CAPEX) option. It is estimated that leased lines account for roughly 15 percent of typical network operating expenditure (OPEX). Wireless backhaul can be more cost-effective than leased T1/E1, DS3, or OC-3 lines. In addition to the economic benefits of ownership, wireless backhaul also allows service providers to retain end-to-end control of their data, gaining the security, stability and freedom associated with full control over their own network. For less populated rural areas, where the cost to lay fibre can be prohibitive, wireless backhaul may be the only viable solution. Finally, because radio propagates over the air faster than light travels through fibre, wireless backhaul can achieve lower latency than fibre.

The purpose of this article is to describe the cooperation between CSIRO and EM Solutions to develop the world's fastest microwave backhaul radio, over the longest link distances, with the lowest latency. This radio was developed for an application in the US financial markets, and achieved 5 Gbps backhaul speeds on links up to $25 \mathrm{~km}$ with latencies of the order of one to two microseconds per hop over the free space propagation delay. Such results are several times faster and longer than other commercially available microwave backhaul solutions, which (at the time of writing) typically have processing latencies measured in tens of microseconds and maximum throughputs of 1 or 2 Gbps, over link lengths approximately one-half of those achieved here for the same availability. 


\section{The Technology Challenges}

As noted above, the key design challenges are to achieve a state-of-the-art data rate, while maximising path length, minimising latency, and maintaining antenna pointing.

Multi-gigabit mm-wave communications systems are commercially available. Typical $\mathrm{mm}$-wave bands suitable for wireless backhaul application include the $60 \mathrm{GHz}$ band and 70/80 GHz E-band. Commercial point-to-point links in the $60 \mathrm{GHz}$ band with data rates of up to 1.25 Gbps are sold by several manufacturers. However, high propagation loss due to oxygen absorption in this band and regulatory requirements limit the communication range for outdoor applications to at most $0.5^{-0.8} \mathrm{~km}$.

The recent availability of the E-band spectrum worldwide provides an opportunity for line of sight (LOS) links with longer range and higher data rates, ideally suited for fibre replacement and backhaul applications. The merits of E-band wireless communications include the vast, uncongested and inexpensive spectrum, where a total of $10 \mathrm{GHz}$ of available $\mathrm{RF}$ bandwidth enables very high data rates beyond $10 \mathrm{Gbps}$ and the use of small, highly directional antennas. In some countries, contiguous bandwidth of up to $4 \mathrm{GHz}$ is available at E-band. Current commercial solutions provide low output power at relatively low infrastructure cost. Current commercial suppliers include BridgeWave, LOEA, Proxim, E Band Communications, Elva, Siae and Huawei. These E-band links are ideally suited for short range (1-3 km for all practical purposes) fibre-quality wireless communications. At the time of writing, solutions up to 2 Gbps using higher order modulation schemes (64QAM) (Huawei 2012) are reportedly available, but these require strong signal to noise ratios (SNR). In more common use are the simpler but more robust modulation techniques, such as amplitude shift keying (ASK) or binary phase shift keying (BPSK) with spectral efficiencies below one bps/Hz, but which only achieve lower data rates. Link distances are rarely more than a few $\mathrm{km}$, in the absence of rain.

Technologies enabling much higher data rates (up to $24 \mathrm{Gbps}$ ) were developed and reported several years ago by the CSIRO ICT Centre (Huang et al 2012a : 122-129) (Huang et al 2011 ). The key algorithms developed (Zhang et al 2012 : 589-599; Huang et al 2012b : 2113-2122) are applicable to systems where the radio channel bandwidth is greater than the Nyquist bandwidth of the associated analogue-to-digital converter (ADC) and digital-to-analogue converter (DAC). Such systems can be utilised for E-band full-duplex wireless links, and can achieve a spectral efficiency scalable from 2.4 to $4.8 \mathrm{bit} / \mathrm{s} / \mathrm{Hz}$ using 8 -phase shift keying (8PSK) to 64QAM modulation, enabling data rates from 12 to $24 \mathrm{Gbps}$. This has been proven by experimental results on a 6 Gbps prototype that achieved a spectral efficiency of 2.4 bps/Hz (Dyadyuk et al 2007: 2813-2821). 
Since the first report, mixed signal processing technologies, higher speed ADC and DACs, and larger scale FPGA devices are now available, and the spectral efficiency of the E-band system above can be further improved. Combined with dual polarisation, a high speed E-band link of up to 50 Gbps data rate can be achieved in $5 \mathrm{GHz}$ bandwidth. However, such data rates have not yet been demonstrated.

Because radio waves propagate through air faster than light travels through fibre, wireless links can achieve lower end-to-end latency. This makes E-band wireless links the medium of choice compared with fibre networks for applications where latency is a concern, such as high frequency trading. Even though the range per hop of the E-band link is still limited, the end-to-end latency of a multi-hop E-band system can be still lower than for fibre if the processing delay at each radio relay node remains small. In mission critical cases, where fibre links can be used as a backup for adverse weather conditions, the per-hop distance of an E-band link can be extended to over $20 \mathrm{~km}$. This will further reduce the overall end-toend latency and deployment cost for the multi-hop E-band link.

The classic way of increasing link range is to maximise the link budget. The link budget is essentially the signal transmit power increased by the sum of receiver and transmitter antenna gains, reduced by the signal to noise ratio required at the receiver to achieve the desired bit error rate at the modulation and bandwidth necessary for the required data throughput.

Increasing the transmit power is an obvious first choice. The most common commercial E-band power amplifiers achieve saturated output powers of $100 \mathrm{~mW}$, but output powers of $1 \mathrm{~W}$ are now becoming available. Changing to a $1 \mathrm{~W}$ power amplifier can theoretically increase the link margin by up to 10dB. However, it is linear power, not saturated power, that is critical in radio communications. In addition, more complex modulation schemes (to achieve a higher bit rate within the same bandwidth) require greater linearity to preserve symbol amplitude than do simpler schemes such as BPSK or QPSK. This means the average transmit power is usually considerably backed-off from the headline saturated power.

The other large variable in the link budget is the antenna gain - doubling the diameter of the antenna increases the link budget by $6 \mathrm{~dB}$ per end, or $12 \mathrm{~dB}$ for each hop. The most common E-band antennas are $300 \mathrm{~mm}$ in diameter; therefore using a $1200 \mathrm{~mm}$ antenna achieves a significant improvement in link budget and consequently range. Unfortunately, a $1200 \mathrm{~mm}$ E-band antenna will have a 3-dB beamwidth of just 0.25 degrees, a pencil-thin beam. This makes it impossible to manually align two ends of such a link several $\mathrm{km}$ apart, or to maintain such alignment when the antennas and their towers are subjected to wind or thermal stresses that arise during normal operation. 


\section{Commercialisation of a long-range, low latency, high speed E-band radio}

With a lead customer in the financial industry requiring a high speed (upgradable to 10 Gbps) low latency radio that could complement a fibre network and achieve a per hop span of $25 \mathrm{~km}$, EM Solutions and CSIRO together developed a radio solution that was able to meet the required specifications. These are summarised in Table 1 below.

Table 1 - Target backhaul E-band radio specifications

\begin{tabular}{|c|c|}
\hline Frequency & E-band (70 to $86 \mathrm{GHz}$ ) \\
\hline Throughput & 5 Gbit/s full duplex \\
\hline Data Interface & 10 GbE Optical SFP IEEE 802.3ae compliant \\
\hline Latency: & $\begin{array}{l}\text { Significantly less than } 1-2 \text { microsec per link end to end } \\
\text { (one hop), excluding propagation delay }\end{array}$ \\
\hline $\begin{array}{l}\text { Minimum operational system } \\
\text { budget }\end{array}$ & $\begin{array}{l}\text { Gross system budget exceeding } 192 \mathrm{~dB} \text { assuming a } \\
\text { minimum receiver SNR requirement of } 9.5 \mathrm{~dB}\end{array}$ \\
\hline Overall BER & $10^{-11}$ per hop \\
\hline Pointing and Tracking & $\begin{array}{l}\text { ISM K-band for tracking compliant with US FCC } \\
\text { regulations, to maintain optimal lock up to a physical } \\
\text { antenna angular deviation of }+/-7 \text { degrees per end }\end{array}$ \\
\hline $\begin{array}{l}\text { Network Management System } \\
\text { interface }\end{array}$ & $\begin{array}{l}\text { SNMP based messaging interface via Ethernet port from } \\
\text { each radio }\end{array}$ \\
\hline
\end{tabular}

CSIRO's focus was on the radio modem. A number of novel techniques were developed in order to achieve high power efficiency and low latency. One major architectural difference with modern lower frequency digital radios was the use of analogue in-phase and quadrature (I/Q) modulation at the IF stage. Using the entire channel bandwidth of over $4 \mathrm{GHz}$ as a single channel presented a number of technical difficulties, particularly relating to maintaining quadrature and gain flatness across the band. This could be estimated and compensated. Other novel techniques included advanced channel estimation and equalisation, algorithmic-efficient transmitter and receiver filter design and implementation, and overall system optimisation to achieve both low latency and high performance at the same time. This required a tradeoff between the number of processing loops involved with channel estimation and the improvement in signal-to-noise ratio that was achieved, against the desired processing latency.

Compared with other commercially available high speed E-band radios, CSIRO's low latency E-band system achieved some distinctive advantages. First, it has low processing latency due 
to the smart signal processing architecture and signalling protocol, even though complicated error correction coding, equalisation, and practical impairment compensation algorithms are employed. Second, it can achieve 10 Gbps data rate with relatively low level modulation (QPSK and 16 QAM) so that it offers higher power efficiency and hence longer distance than more complex modulation formats. Third, with its advanced channel estimation and equalisation techniques, it can cope with harsh channel conditions with tens of nanoseconds delay spread caused by analogue circuitry, cable reflection, and multipath propagation. Finally, it offers flexible switching between Ethernet traffic and ultra-low latency relay traffic and among multiple radios, so that a multi-hop E-band link can be configured with fibre backup, suitable for low latency application under all weather conditions.

EM Solutions' focus was on the commercialisation and packaging of the radio modem itself, and the design and manufacture of the antenna and feed, the automatic pointing system and the overall network element management system. Although the management system was straightforward, design of an antenna pair that would automatically maintain alignment along the boresight between both ends proved a challenge. Tower vibration of even a fraction of a degree, for instance due to wind or thermal variation, will twist the antennas to the extent that communications would be impossible between them. One solution would be to use a phased-array antenna with electronic beam steering. Conceptually simple, such a solution was considered too costly to develop from scratch. The approach we adopted was to mount a high-gain parabolic antenna on a two-axis gimbal system, with low friction contactless motors mounted within the bearings to drive the antenna in either the azimuthal or elevation direction as required to maintain boresight. The resulting radio is shown in Figure 2. 


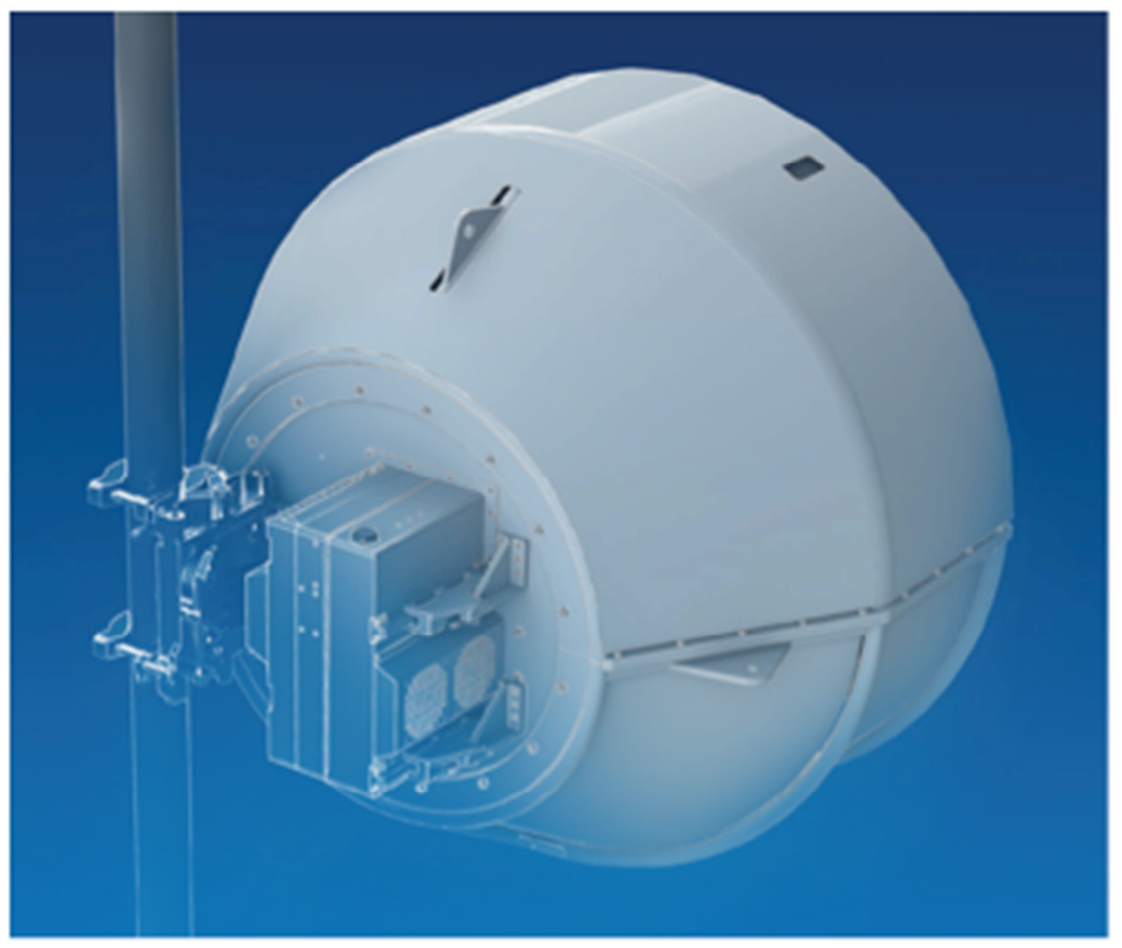

Figure 2: The commercialised E10G radio, with the $1.2 \mathrm{~m}$ antenna and gimbal system housed with a protective shroud. Portion of the automatic pointing control system and radio modem can be seen mounted at the rear of the antenna.

Determining the required direction to maintain alignment borrowed an idea from radar technology, a technique known as monopulse. This uses a broad beamwidth signal (a beacon) emanating from the remote end, and measures the phase difference across two slots either side of the central feed at the near end antenna. If the near end antenna is pointing directly towards the far end beacon, the phase difference will be zero. The phase difference will gradually increase as the antenna is pointed off-boresight. With two pairs of slots along a horizontal and vertical axis, a vector to indicate the centre of boresight can be generated from these phase measurements, and used to drive the motors to re-centre the antenna along boresight (where the phase error will be zero, and the received beacon and data signals a maximum).

Because for initial acquisition the beacon signal from the far end needs to be of broad beamwidth to fall within the capture angle of the near antenna, it cannot be the E-band data signal itself, since that is too narrow in beamwidth (0.25 degrees for a 1200mm antenna). In the EM Solutions' system, the acquisition beacon was generated at K-band by a second colocated antenna at each end, a horn with a broader beamwidth and low enough gain to meet the radiated power regulations of the band, so that it illuminated the receiver end regardless of its own vibration and in whatever direction the near end or far end antenna is pointing (within reason). A very narrowband tuned receiver is able to detect the transmitted signal since the frequency is known according to a defined frequency plan. A concept is shown in 
Figure 3. Such a system proves remarkably effective at acquiring the remote end in a matter of seconds and maintaining lock even when the antennas at either end were violently shaken. This should be compared with fixed high gain antennas that can otherwise take hours to manually align using optical telescopes and maximum signal strength indicators, and that have no resilience to any small motion at either end.

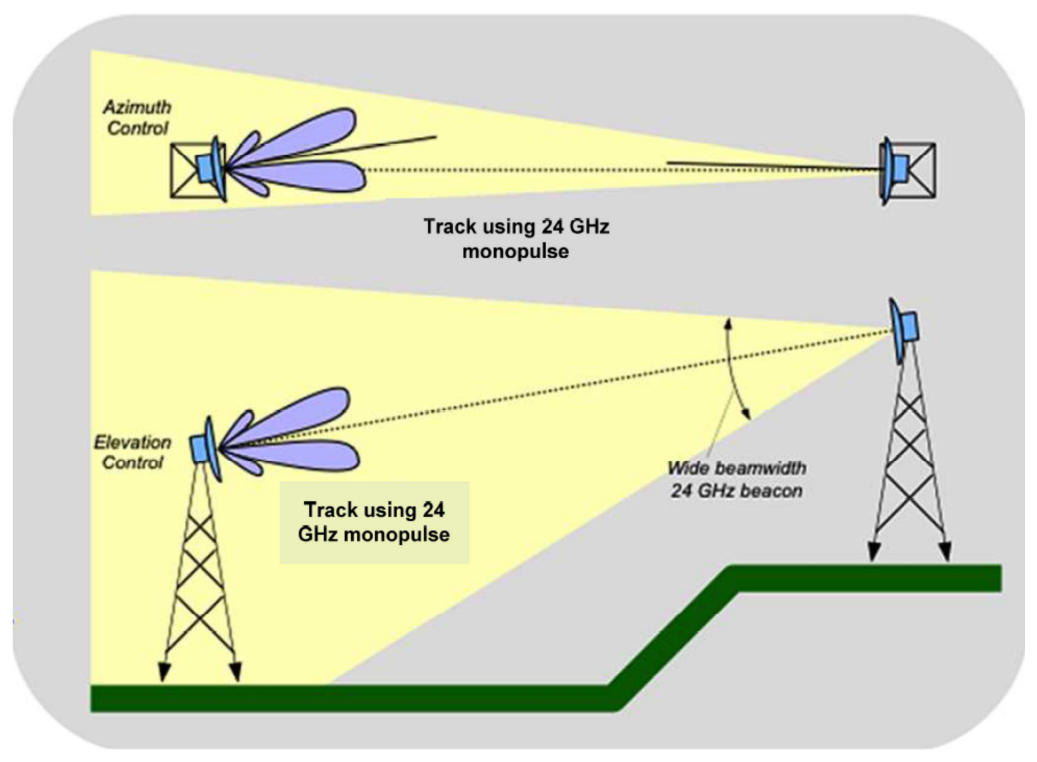

Figure 3: The concept of pointing using a K-band beacon transmitter at the remote end for monopulse detection at the near end, to steer the near end antenna back to boresight.

\section{Field Results}

The system was installed in a range crossing the upper reaches of the Brisbane River, between a University of Queensland site on the outskirts of Brisbane, at Pinjarra Hills, with line of sight visibility to a site $16 \mathrm{~km}$ away owned by the Bureau of Meteorology at Springfield, near Ipswich. The system was transported using a special frame on a flat-bed truck. Lifting, placement, and tie down took no longer than 20 minutes. Lifting was simplified because the unit is neutrally balanced, and fitted with side hooks for steering lines. The antenna and its pointing system can be mounted to a standard $120 \mathrm{~mm}$ pole, using either a low weight normal-strength or high-strength mounting bracket. Figure 4 shows the unit being lifted and installed in place. 

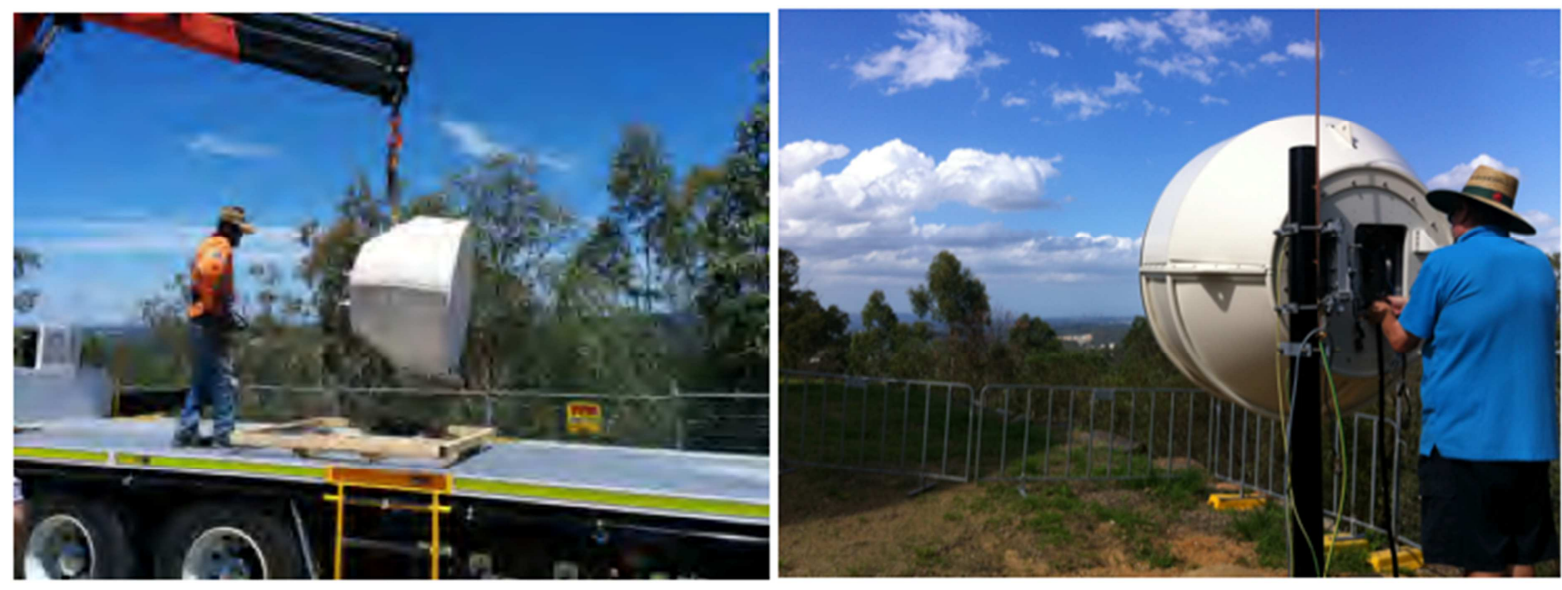

Figure 4: (a) One end of the E-band radio being lifted for mounting and (b)the radio installed at one end of the trial link.

Removal of the gimbal packing and fitting of the radome takes a further 20 minutes, and the electronics modules are then attached to the rear of the unit using hex keys within a similar period of time. Three cables are required, the first for the optical data payload, the second for optical control and monitoring, and the third to provide 48V DC power. Power up and initial scan takes approximately 2 minutes, and requires only coarse pointing towards the far end (which is barely visible). As the system automatically acquires the remote end, feedback on the pointing response, off angle error, bit error rate, and received signal strength is achieved through the user interface.

Upon start-up, each end searches for the wide angle beacon signal emanating from the other end. Each system then locks on to the beacon and moves to closed-loop pointing control. At this stage, depending on the motion at the near end, the system begins the search for a narrow beam, higher strength beacon signal that is transmitted using the main antenna reflector at the far end, rather than the low gain horn. This beacon signal is more directional than the first that was used for initial acquisition, and can assist general pointing performance due to its higher beacon signal to noise ratio, as well as in cases where multipath propagation might affect the broader beamwidth beacon. Gyroscopes that measure the acceleration along the two axes of the antenna are also used in a feedback loop to adjust the pointing direction.

Once the system has optimised its pointing, data communications at E-band can begin. Pointing accuracy was measured in real time to be within 50 millidegrees of true boresight. The QPSK constellation shown in Figure 5 was observed to be clear and achieved bit error rate less that $2 \mathrm{E}-13$ for carrier to noise ratios better than $9.5 \mathrm{~dB}$ consistently over a path in excess of $15 \mathrm{~km}$. 


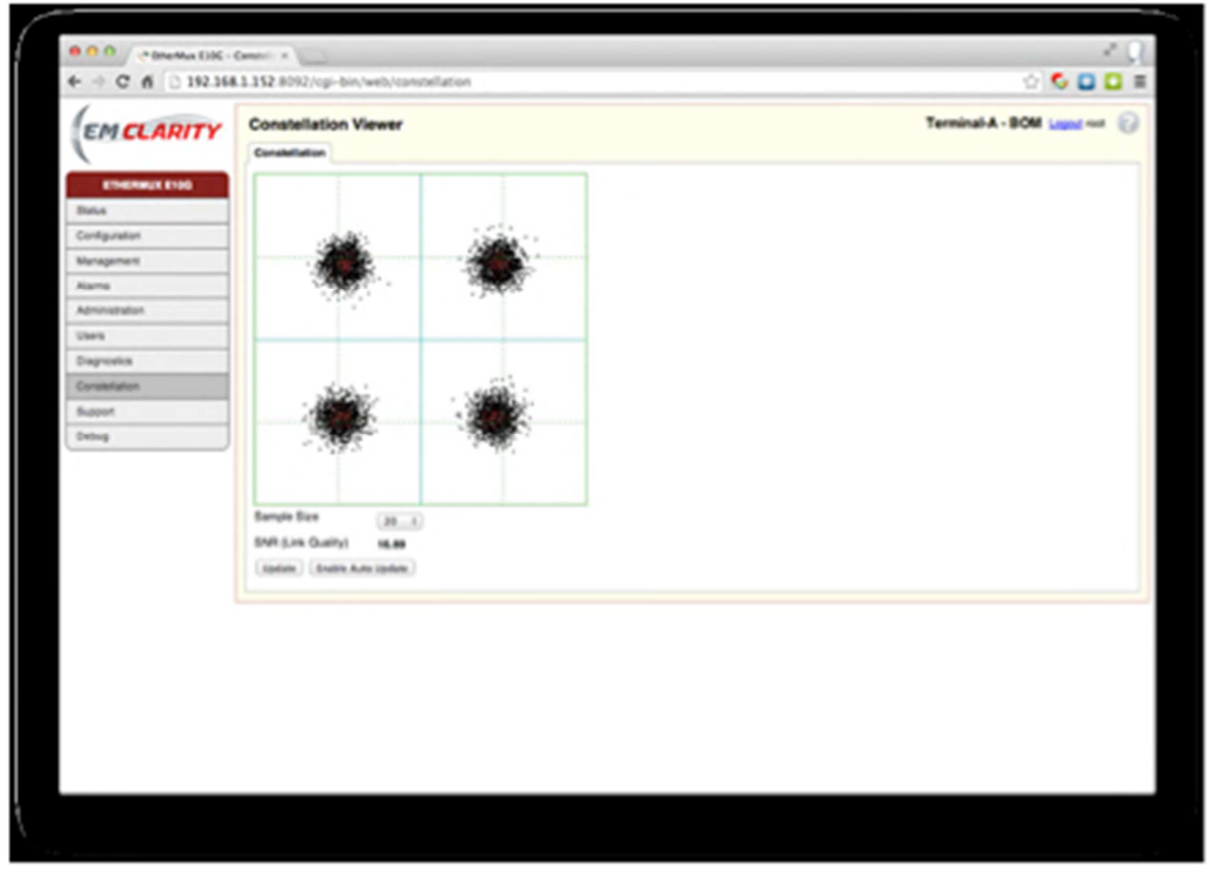

Figure 5: Constellation diagram of the received QPSK signal after downconversion from E-band to baseband.

The link was left running for a total of 30 days in various types of weather. Outages occurred during thunderstorms, since heavy rain is essentially impenetrable at E-band for distances exceeding $5 \mathrm{~km}$ (for this system; and $1 \mathrm{~km}$ for other commercial systems). For example, on December 19, a typical summer day, the link margin was measured to be $17 \mathrm{~dB}$ when the temperature was 27 degrees and humidity $43 \%$. On December 12, when the humidity was greater than $90 \%$, the link margin had dropped to $11.9 \mathrm{~dB}$ over the same $16 \mathrm{~km}$ path length (the $\mathrm{e}^{-11}$ BER threshold SNR of the system is $9 \mathrm{~dB}$ when running QPSK). In both cases, no bit errors were observed over a 24 hour period with data passing at a throughput of $5 \mathrm{Gbps}$ over the link. Figure 6 shows the recorded SNR and RSSI and other performance parameters as a function of time. 


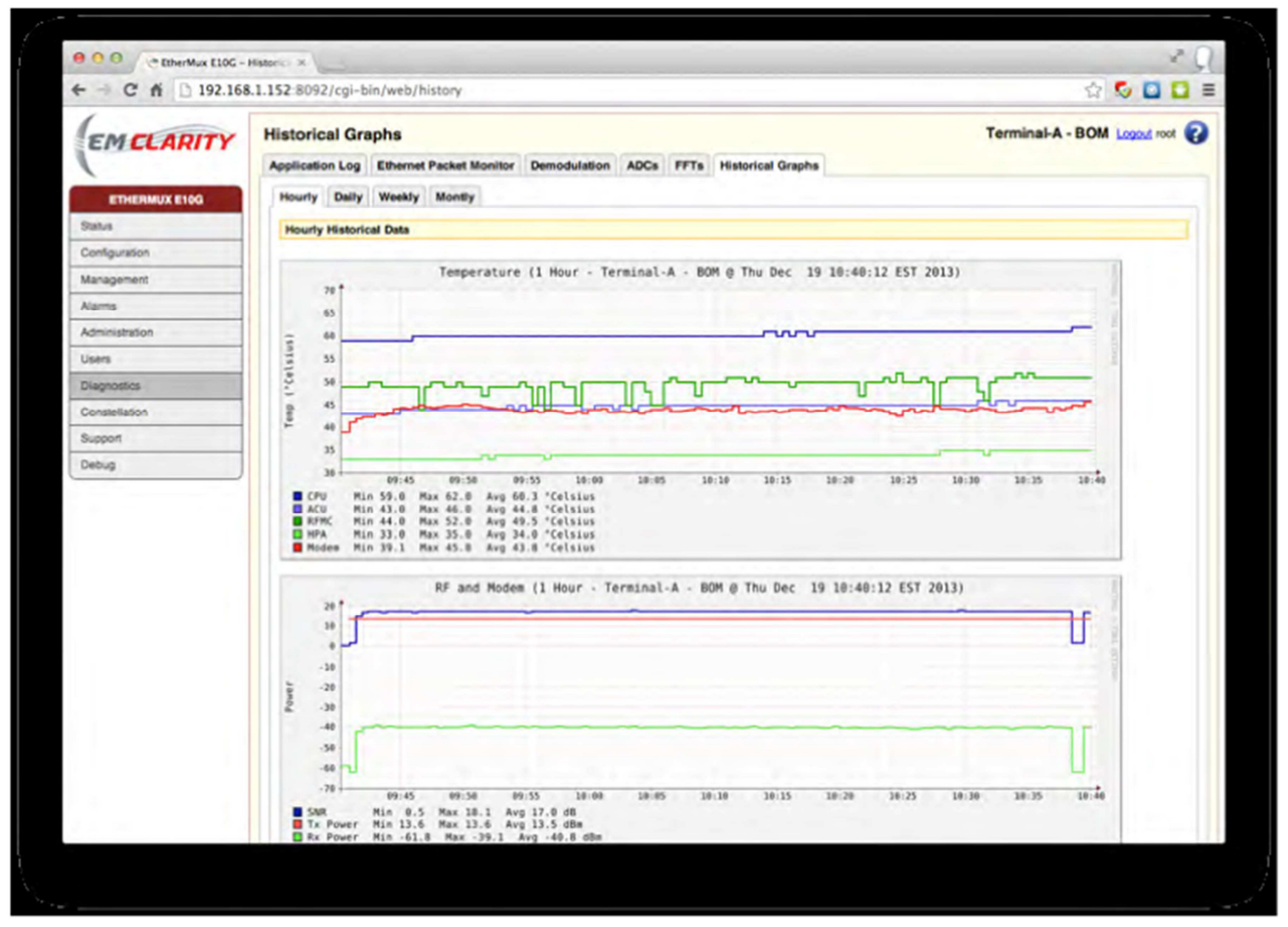

Figure 6: A performance log of the system during field trial. The upper traces show temperature of critical system components and the lower traces show SNR, Tx power and Rx power during a typical day of testing.

Latency could not be measured in the field since its measurement requires a loopback, and this would include a long and potentially variable propagation time over the transmission path. Instead, a known length of fibre cable was used for testing in the lab, and latency tests were conducted on the inter-link connection, with a latency measured to be well under two microseconds. The latency from the 10Gig Ethernet input port to the link side of the radio was also measured to be similar for small packets.

\section{Comparison with Other Technology Options}

There are a number of alternative ways to achieve further improvements in the data rate, such as employing higher order modulation, making use of multiple input multiple output (MIMO) antennas, improving link availability, and increasing transmit power.

The highest order modulation reported in a commercial microwave product is 1024QAM. Due to the DAC/ADC speed and resolution, as well as phase noise issues, such higher order modulation is possible only for narrow bandwidth (below $100 \mathrm{MHz}$ ) systems.

For wider bandwidth systems in mm-wave bands, the highest modulation so far reported is 64QAM. Employing high order modulation such as this incurs some system penalties, such as design and implementation cost, and reduced receiver sensitivity i.e. higher signal-to- 
noise ratio (SNR) requirements, as well as reduced output power due to higher linearity requirements for the power amplifier. This reduction in both transmit power and receiver sensitivity will of course result in reduced link distance.

The use of line of sight (LOS) MIMO in microwave backhaul has been recently demonstrated by Ericsson. By combining a 2x2 LOS MIMO (2 receive and 2 transmit antennas relying on path diversity) with dual polarisation and 1024 QAM modulation, a spectral efficiency of $36 \mathrm{bps} / \mathrm{Hz}$ can be achieved, yielding $1 \mathrm{Gbps}$ throughput in a $28 \mathrm{MHz}$ channel (Hansryd et al. 2011). This requires two radios at each end. A similar announcement has been made by MIMOtech with its Starburst Janus, an ultra-high capacity packet radio for last mile backhaul, which utilizes a 4x4 LOS MIMO yielding a spectral efficiency of $25 \mathrm{bps} / \mathrm{Hz}$ (Microwave Journal 2013)(Cellular 4G/LTE Channel/Industry News 2013). As a promising technology for future high speed wireless communications, LOS MIMO has also been proposed by the US Defense Advanced Research Projects Agency (DARPA) in its 100G program (DARPA-BAA-13-15) to develop a 100 Gbps RF backbone using E-band mm-wave frequency spectrum.

At E-band, any quoted operational distance is complicated by its relationship with link availability. Achieving high link availabilities, of the order of $99.99 \%$ or higher as required for critical telco links, normally entails very short hops, to overcome the effect of rain and humidity that may (rarely but occasionally) extend across the entire link and prove impenetrable to the signal. Thus hop lengths in the tropics must be shorter than those in the desert to achieve the same availabilities, even though the radio systems are identical. Use of automatic transmit power control to increase transmit power to the maximum level during a rain fade can allow the link to overcome the fading effects. Other techniques include adaptive coding and modulation (ACM) and adaptive rate (AR). Such schemes change either the modulation scheme or the channel bandwidth in response to worsening path loss. For mmwave radios which only operate with low order modulations, AR may be a better solution. By taking advantage of the large available bandwidth, AR can keep the modulation constant but reduce the transmitted symbol rate and thus the data rate. This reduces the bandwidth, and hence the noise floor to improve the signal to noise ratio during rain fades.

Increasing transmit power is the most straightforward way to increase the link distance. However, this may create both regulatory and technical issues. Although lightly licensed, depending on the country of operation E-band transmission still requires satisfying Australian ACMA or United States FCC rules (ITU Region 3 or 2 respectively) with respect to radiated power. Technically, trying to increase power amplifier output power continues to be one solution for microwave systems. For mm-wave systems, using antenna arrays with beamforming will be another potential solution, where each individual antenna element can 
have low power, but as a spatially combined system of many elements the total emitted power can be high. Challenges include MMIC integration, devising an efficient digital beamforming algorithm, and compensation for mutual coupling between elements. Adaptive beamforming (Guo et al. 2010; Huang et al. 2010 : 1770-1779) and electronic steering are promising future research directions to achieve even longer range inter-aircraft, aircraft-tobase station, and aircraft-to-vehicle communications.

\section{Conclusion}

With the advance of broadband wireless access and next generation mobile systems, backhaul infrastructure is being stressed by demand for higher data rates. As cost-effective alternatives to fibre, high speed and long distance wireless backhaul is becoming increasingly attractive. However, there are significant technical challenges such as achieving higher spectral efficiency and extended transmission range.

This article has described an E-band mm-wave backhaul radio modem coupled with a high power amplifier and an automatic pointing system to enable the use of $1.2 \mathrm{~m}$ antennas at the end of each hop. This system achieved data rates of 5 Gbps over link distances of up to $25 \mathrm{~km}$, and maintained communications even under severe vibration of the tower due to wind effects. The measured latency was significantly less than two microseconds. This system resulted from a collaboration between CSIRO and EM Solutions, and resulted in the world's fastest commercially available radio, with the highest link budget for the data throughput, to achieve the longest link distances, and with the lowest latency.

\section{References}

Cisco. 2014. "Cisco Visual Networking Index: Globe Mobile Data Traffic Forecast Update, 2014-2019," white paper, available at:

http://www.cisco.com/c/en/us/solutions/collateral/service-provider/visual-networkingindex-vni/white paper c11-520862.html

Dyadyuk, V; Bunton, J; Pathikulangara, J; Kendall, R; Sevimli, O; Stokes, L; Abbott, D. 2007. "A Multi-Gigabit mm-Wave Communication System with Improved Spectral Efficiency". IEEE Transactions on Microwave Theory and Techniques, vol. 55, no. 12, pp. 2813-2821.

Fodor, G; Dahlman, E; Mildh, G; Parkvall, S; Reider, N; Miklós, G. 2011. "Evolution of LTE toward IMT-Advanced," IEEE Communications Magazine, vol. 49, no. 2, February 2011, pp. $84-91$.

Fu, IK; Chen, YS; Cheng, P; Yuk, Y. 2010. "Multicarrier Technology for 4G WiMAX System," IEEE Communications Magazine, vol. 48, no. 8, August 2010, pp. 50 - 58.

Guo, Y. J; Bunton, J; Dyadyuk, V; Huang, X. 2010, "Multi-Stage Hybrid Adaptive Antennas," Australian Provisional Patent, AU2009900371 (2 February 2009), PCT published (20 August 2010) WO 2010/085854 A1. 
Hansryd, J.; Edstam, J; “Microwave Capacity Evolution,” Ericsson Review, June 2011, available:

http://www.ericsson.com/news/110621 microwave capacity evolution 244188810 c

Huang, X; Guo, Y. J; Bunton, J. 2010. “A Hybrid Adaptive Antenna Array,” IEEE Transactions on Wireless Communications, vol. 9, no. 5, May 2010, pp. 1770-1779.

Huang, X; Murray, B; Bunton, J; Guo, Y. J; Dyadyuk, V. 2011. "Wireless Data Communications". Australian Provisional Patent (AU2009903399P) (July 2009), PCT published (WO 2011/o09157 A1

Huang, X; Guo, Y. J; Zhang, J; Dyadyuk, V. 2012a "A Multi-Gigabit Microwave Backhaul," IEEE Communications Magazine, vol. 50, no. 3, March 2012, pp. 122-129.

Huang, X; Guo, Y. J; Zhang, J. 2012b. "Sample Rate Conversion Using B-Spline Interpolation for OFDM Based Software Defined Radios," IEEE Transactions on Communications, vol. 60, no. 8, August 2012, pp. 2113-2122.

Huawei. 2012. "Huawei Debuts 2nd-Generation Ultra-Broadband E-Band Microwave," Press Release, 2 October 2012; availableat : http://www.huawei.com/en/abouthuawei/newsroom/press-release/hw-194598-e-band microwave.htm

Microwave Journal. 2013. Cellular 4G/LTE Channel/Industry News. 2013. "MIMOtech Launches Ultra High Capacity Radio for Last Mile LTE Backhaul," Microwave Journal, 20 May 2013, available at: http://www.microwavejournal.com/articles/19885-mimotechlaunches-ultra-high-capacity-radio-for-last-mile-lte-backhaul

Transmode. nd. "Low Latency - How low can you go?” white paper, available at: http://www.transmode/en/ resources/whitepapers

Zhang, J; Huang, X; Cantoni, T; Guo, Y. J. 2012. "Sidelobe Suppression with Orthogonal Projection for Multicarrier Systems," IEEE Transactions on Communications, vol. 6o, no. 2, pp. 589-599. 